$\xi=-1$

\title{
Timber robotic fabrication: testing for an integral manufacturing
}

\author{
Chadi El Khoury *, Marwan Halabi \\ Assistant professor, Faculty of Architecture-Design \& Built Environment, Beirut Arab University \\ *Corresponding author E-mail: s.khoury@bau.edu.lb
}

\begin{abstract}
Going beyond sustainability and with the great development of technology, timber has a great potential to be explored as a building material. Its physical-mechanical properties such as lightness and elasticity allow the designing of complex structures and the growing trend in research in robotic fabrication has accelerated the development of dimensional design concepts that demonstrate that wood is absolutely contemporary and at the height of other innovative materials. This paper investigates on computer aided integrated architectural design and production of timber using advanced automated tools, aiming to provide integral solutions for the design and production of geometrically complex free-form architecture. Investigations on computer aided geometric design and integrated manufacturing are carried out with equal importance. This research is considering an integral and interdisciplinary approach, including computer science, robotics and architecture. The studies for translation of the geometrical into constructional elements consider integrated manufacturing. Addressing and numbering of the elements by iterative geometric design are investigated and compared to lexicographically ordered addressing systems, in order to provide an adequate data structure for the design, production and assembly of the constructional elements. The integrated digital design methods studied are tested and verified by the realization of one to one scale prototypes. The main aim of the research is to find ways of shifting the perspective of adventurous high quality architecture robotically produced with correspondingly reduced costs and minimized environmental impact.
\end{abstract}

Keywords: Integral Manufacturing, Parametric Architecture, Robotic fabrication, Timber,

\section{Introduction}

For a long time the construction of wood has been governed by the use of linear elements connected to trellis systems. However, engineers and architects have done little to develop the use of wood as building material unlike reinforced concrete that has predominantly become one of the building materials with the greatest mechanical and functional potentials in architecture.

Already in the 60's and 70's of the last century, the debate on the environmental issue emerged with the formation of the first Environmentalist Associations, with the aim of defining a central link between economy and environment in the obvious need to preserve the quality of the natural heritage, in addition to reviewing and balancing development models. These were the years paving the way for profound debate and growing attention from the scientific community and civil society to consider the planet as a closed system in which every natural resource finds its limits in the availability and capacity of absorption of the ecosystem, thus taking into account the limits of development.

The conversion of purely geometric figures into applications in the field of architecture poses a certain number of questions. As a statement, a vast proportion of virtually designed geometries do not correspond to the constraints emanating from the different construction processes in architecture [1]. Designed geometric objects of certain complexities such as the double curvature make their physical realization difficult. The production of double curved elements is time intensive and its costs remain relatively expensive, despite the use of integrated manufacturing techniques. A certain number of points aiming to optimize the production costs is already integrated in the constraints, which the digital model is capable of verifying [2]. Further optimization tries to close the gap between the early design stage at the computer screen and the physical production, be it manual or automated. Generally, the geometric object does not meet all properties that are necessary for its physical construction [3]. Therefore, several post-processing methods will have to be applied in order to translate the geometric data into a coherent set of constructional strategies of elements. These post-processing methods can be split into the following work steps:

- Computation of the geometric data of the constructional elements;

- Mapping functions relating data from the geometric figure to the constructional elements;

- Automated creation of machine instructions for integrated manufacturing;

- $\quad$ Addressing and labeling of the constructional elements for facilitating the logistics during both production and assembly.

The application of iterative geometry for architectural use is central to this work. It provides a way to verify the validity of the established methods, with the range of potential applications reaching from purely decorative architectural elements, representing an ornamental character, to big scale structures, which provide load-bearing capacities [2]. The applications are tested on a series 
of reduced scale prototypes, which verify the suitability of iterative geometric design for architectural use.

\section{Kuka robotic arm}

Nowadays, a large number of computer numerically control machines are available on the market. They can be distinguished for freedom of movement, cutting tools, machine code, and control software [4].

The cutting method presented in this paper utilizes the Kuka robotic arm with 6-axis planes "Fig.1", already used by some woodworking companies. In addition to the usual three $\mathrm{X}, \mathrm{Y}$ and $\mathrm{Z}$ translational axes, 6 axis enabled machines are equipped with additional cardanic rotary axes defined as A4, A5, and A6, which allow the tool to be oriented longitudinally, which does not need to be perpendicular to the working table (XY plane).

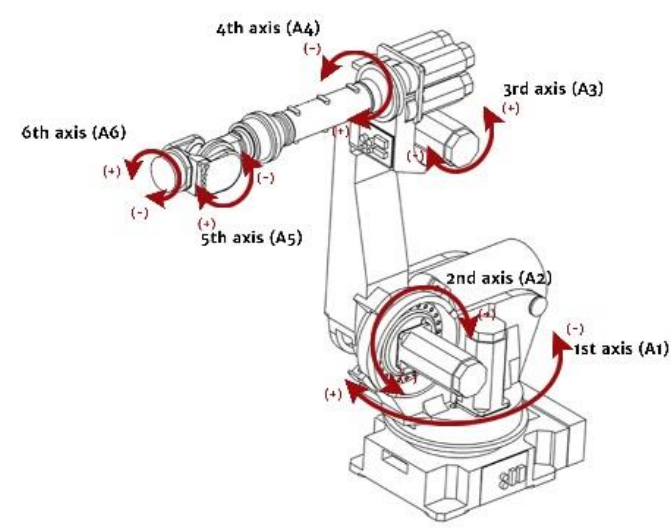

Fig. 1: Basic Scheme of movement of the robotic arm used for the experiments (image from author)

\section{Wavy fence installation}

The wavy fence installation is the first exercise done using the robotic arm, situated in the front of the Digital Fabrication Lab in the faculty of architecture at Beirut Arab University in Debbieh, Lebanon. The simple idea, harmonized with the surrounding landscape, is an iteration of a series of beams of rectangular section of different lengths and angles of solid wood, chosen to be the African Tek wood (Iroko), of a cross section of $50 \mathrm{~mm} \times 100 \mathrm{~mm}$, that is complexified by the shape control potential today possible through the parametric design programs.

The design represents a grooved surface that moves in relationship to orographic constraints and with the kinematic vision of passers. A sinuous screen that reaches a height of 2.4 meters and that passes from a concave to a convex, sometimes permeable to the eye and sometimes opaque depending on points of view, builds a sort of environmental sculpture which identifies the entry to the Digital Fabrication Lab. A layer of white and fine gravel hides the interlocking of beams driven into the ground, hiding the structural detail to pave way for a pure perception of the movement and the play of shadows to vary throughout the hours and seasons "Fig.2".

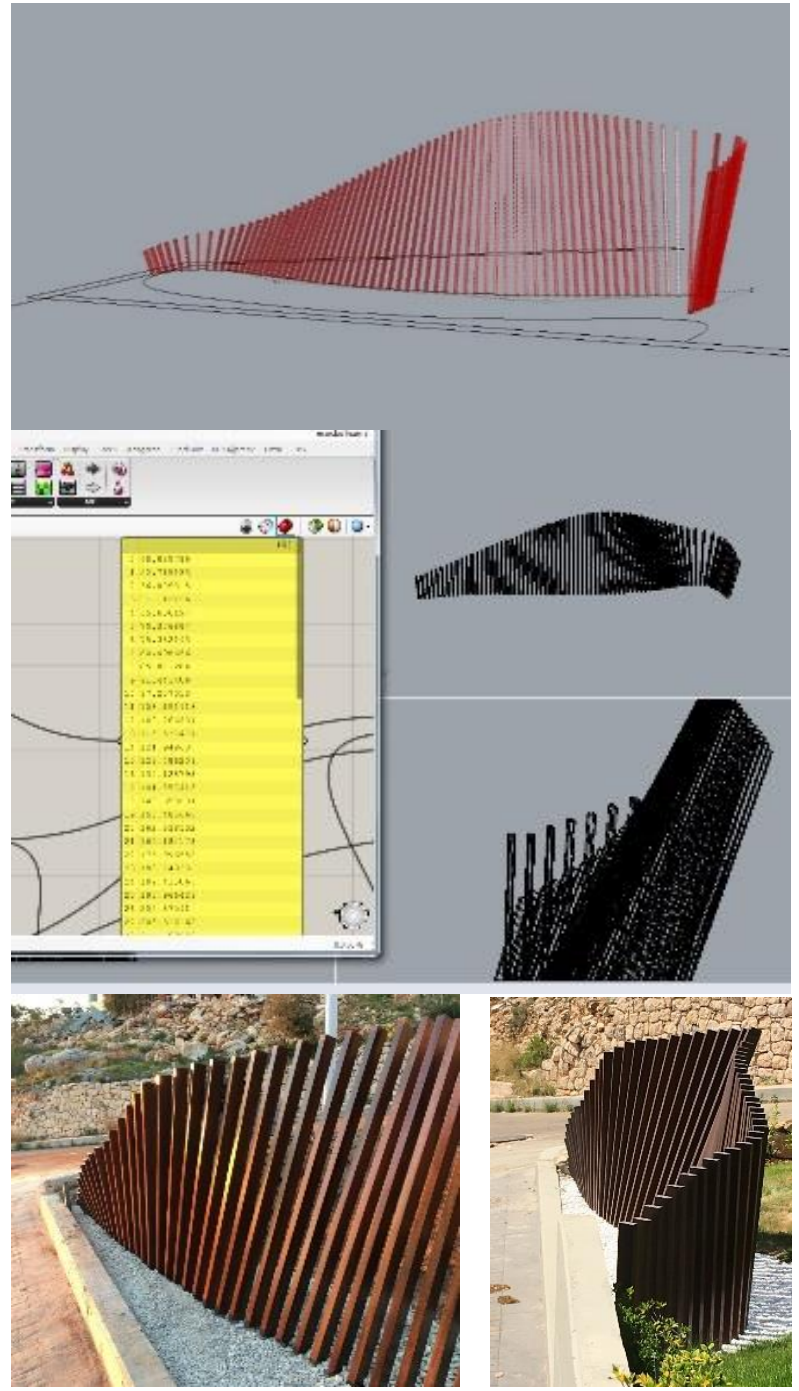

Fig. 2: Design Scheme of the digital model compared to the outcome (images from author)

The shape is guided by two different graphs of curves, a lower and a higher one, in addition to a third curve related to the different angles with positive and negative values. A panel is related to the guiding lines with a distance component that can measure exactly the dimensions of the beams with their proper angles. This procedure was used according to the Gaussian graph.

\section{The cloud and the shadow}

For the second experiment, the basic idea was to design a shading structure that would be installed at the entrance of one of the faculties at the campus. The shape would be composed of a free surface with a combination of hyperbolic and elliptical paraboloids, so to exploit some particular properties of these surfaces. The size of the element was approximately of $10 \mathrm{~m} \times 8 \mathrm{~m} \times 3 \mathrm{~m}$ and supposed to be designed and built in just 7 days. In addition, students were supposed to collaborate in the design and fabrication of 100 textured panels to be installed in the modules of the structure. Once the structure is placed in its final position, the panels will generate a complex drawing of shadows. Considerations were taken in order to use $22 \mathrm{~mm}$ thick plywood panels that are commonly available in a size of $2440 \mathrm{~mm} \times 1220 \mathrm{~mm}$. The fabrication consisted of a series of steps. The first one is the offset, which starts from the poly-surface the faces to be offset automatically by the help of a script. For general planar, quadrangular meshes, the offset in a single vertex is not possible. In this case, a triangular face cuts the corners of the panels so that the vertex in the offset faces is a quadrangle. By choosing a constant inclination angle, a 
guarantee the offset in a single vertex for all faces and adapt the script to this particular situation could be attained.

Later, an address number composed by the line and the row number identified all panels. Concerning the panel layout, components were laid out by lines on twenty two plywood panels "Fig.3". The fact that the basic geometry can be developed as a single, continuous surface helped to organize the faces on the panels with a minimum of waste. This is an important advantage for the economic construction of folded plate structures.

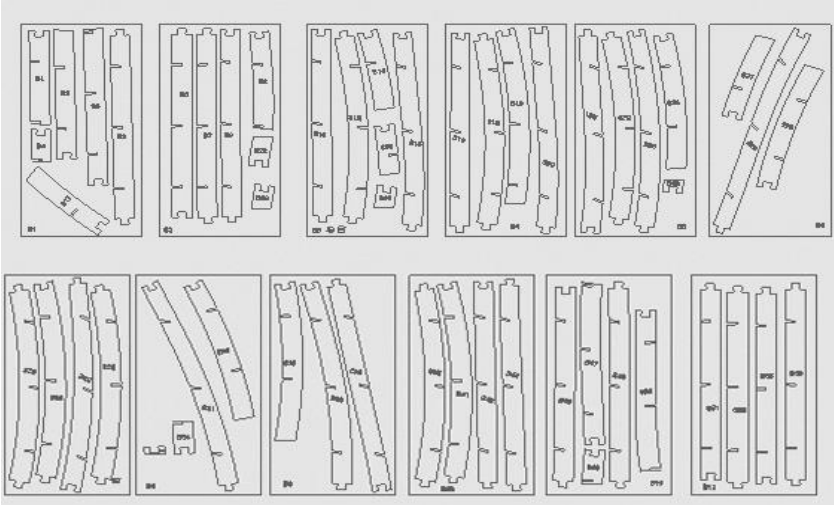

Fig. 3: Panel layout example to be transformed into a g-code (image from author)

The next procedure to set is the tool path. In this case, the order of milling operations and the tool path were generated by a second script. The faces had to be fixed on the machine base so that they do not move when they were cut out of the plywood panel. Therefore two drilling holes were defined in the diagonal of each panel. The position of these holes had to be controlled so that the screws are not in the way of other milling operations. Each side of the faces is milled in three runs. The number of runs is linked to the tool size and the milling speed. Three holes for dowel connections are drilled in the oblique sides. Their position is automatically fixed in function of the length of the sides and in order to keep a minimal distance to the edge. Due to their geometry, the parallel sides are not accessible for the milling tool.

G code for kuka robot

$1 \mathrm{P} 8766268$

2 (***** 17682 commands to process $* * * * *)$

3 (****** masterfile y:/ P8766268_5x_jointing_2014 -3 -6_11 -35 -13 *****)

4 N10 G47

5 N20 T27 M6

6 N30 G47 A0 B0 F8000

7 N40 S13000 M3

8 N50 G49 G55

9 N60 P4010:0 (lower aspiration )

10 N70 (dual infeed mode )

11 N80 G0 X0 Y0 Z70 ( startpos )

12 N90 G0 X945 .959 Y1530 .901 Z66 .308 A109 .306 B -40.447 (safe)

13 N100 G1 X993 .829 Y1547 .671 Z6 .808 A109 .306 B -40.447 F5000

14 N110 G1 X997 .514 Y1539 .217 Z6 .808 A112 .656 B -40.373 F5000

15

16 N260 G1 X1034 .094 Y1464 .454 Z8 .753 A160 .451 B -26.853 F5000 17 N270 G1 X1035 .013 Y1465 .004 Z8 .753 A169 .049 B -23.399 F5000 $18 \mathrm{~N} 280$ (*** turn ***)

19 N290 G0 X1033 .574 Y1457 .57 Z26 .253 A169 .049 B -23.399 (retreat)

$20 \mathrm{~N} 300$ G0 A -179.229 B -20.435 (new ab)

21 N310 G1 X1035 .013 Y1465 .004 Z8 .753 A -179.229 B -20.435 F1500

( back )

$22 \mathrm{~N} 320$ (*** end turn ***)

23 N330 G1 X1035 .932 Y1465 .555 Z8 .753 A -179.229 B -20.435 F5000

24 N340 G1 X1036 .85 Y1466 .106 Z8 .753 A -163.936 B -18.433 F5000

25

26 N176820 G0 X347 .44 Y483 .127 Z66 .308 ( safety )

27 N176830 G0 X0 Y3700 Z70 ( endpos )

28 N176840 M5

29 N176850 M30

30 \#

Fig. 4: G-code generated from the drawing (image from author)
Later, the machine code was generated as a script supposed to translate the tool path into the machine code, which in this case was the g-code "Fig.4". The machine code then was tested $50 \mathrm{~cm}$ below the machine table and with low speed to evaluate its correctness. The faces were milled within a day. Each face is marked by its number.

Concerning the assembling, the faces were assembled horizontally by lines, being joined by dowels, and with some screws added to keep the faces in place. Stickers were used to control the position of the faces during the handling and the transport. The lines have been piled up and connected by dowels drilled with a lamello machine. The folded plate structure has been transported in two pieces that were assembled on site.
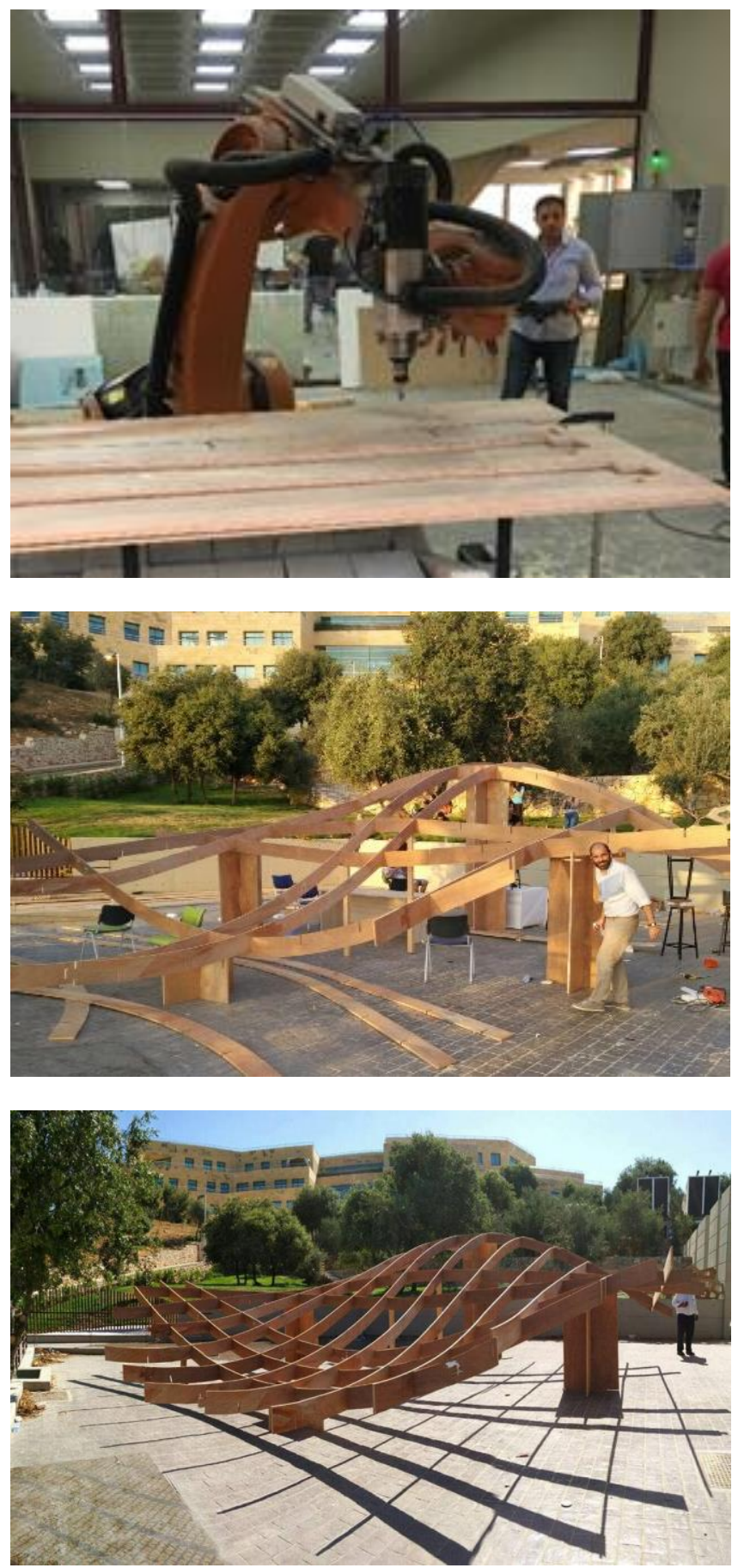

Fig. 5: Fabrication and Assembly of the structure (images from author) 


\section{Robotic fabrication of the structure of a ruled surface}

The idea of working with the form-finding strategy has the potential of fusing digital geometrical principles with material properties of diverse configurations. Physical models were some of Antoni Gaudí's strong line of research throughout his work that used to deal with challenges facing structural and material consistency, in addition to the highly complex aesthetics of the final results. For instance, Gaudí intended to innovate the structural appearance of the Sagrada Família by proposing helical columns of double twist [5].
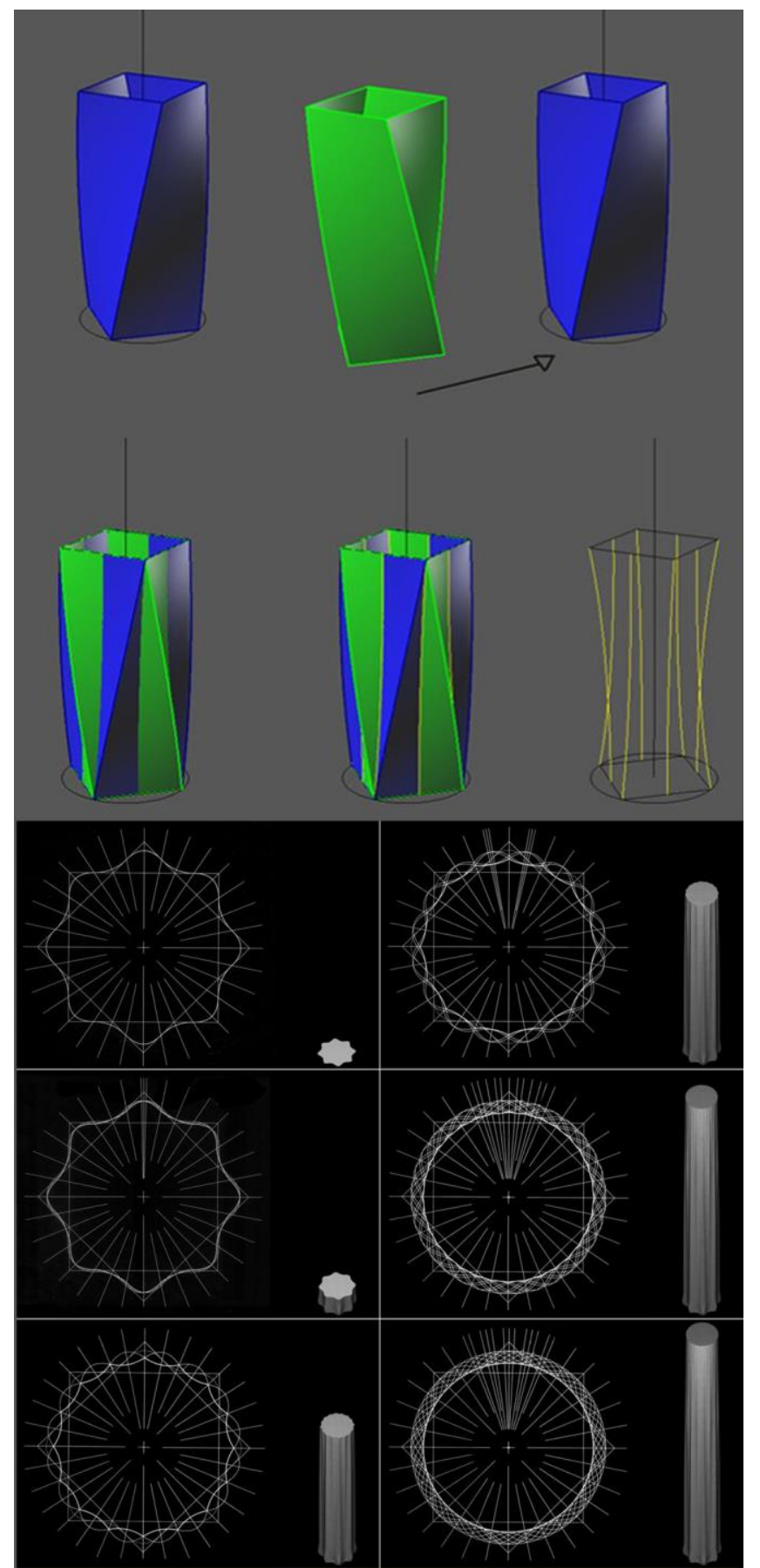

Fig. 6: Design principle of a double twist helical element, where Antoni Gaudí uses the intersections of the column in order to obtain as a result the "new order" (Image from author)

Based on polygons and defined as the hexagon and octagon, the design inscribed in parabolas rounding the corners or vertices of each point of the star "Fig. 6". The Solomonic column could be obtained from this base with a "sweeping" movement, encompassing the helix to the right, and with another column composed from the same base turned to the opposite direction. In a progressive multiplication strategy, Antoni Gaudí built plaster models at 1:10 scale, with intersecting surfaces meeting when turning, according to the number of sides of the polygon, and always turning the same polygon.

To set up a design to fabrication experiment, a pre-defined topology such as a basic ruled surface, related to physical components like standard rectangular cross-section beams were pre-defined as the basic conditions for test. In addition, material assemblies such as wooden plates, chains and strings, coupled with gravity and surface tension were to be taken into consideration as integrated parameters affecting the aesthetic of form, keeping in mind that physical construction using such materials would implicate in time consumption after the fabrication of the main skeleton of the parabolic element.

The hyperbolic paraboloid is a doubly ruled surface containing two families of mutually skew lines [6]. In the digital realm, they are defined as isocurves. The lines in each family are parallel to a common plane, but not to each other. Hence, the hyperbolic paraboloid is a conoid constructed from straight lines.

This property makes easy to build a hyperbolic paraboloid using concrete, and explains its frequent use in modern architecture. The main motivation of this exercise was to improve the productivity and construction quality of a component that can in principle be built either manually or with basic automated tools, however, proposing a construction solution that could only be made robotically if the high precision and optimization of time were major parameters.

Once the paraboloid is defined in space, transforming it in a volumetric entity requires a series of parameters that are relatively easy nowadays to apply to it. However, the geometrical relation between the original and extruded or offset data provides a transformation of their edges, which may imply in the complexification of the fabrication process "Fig. 7".

Depending on the approach of transformation from digital to physical states, a series of steps should be created within the definition of the fabrication strategy. Depending on the final materiality, fabrication can vary from using bi-dimensional elements to complex bent panels to be unfolded, cut and bent. The strategy chosen for the production of the parabolic skeleton was the rectangular cross section timber beams that would be connected by wooden hooks and with the slots robotically fabricated.
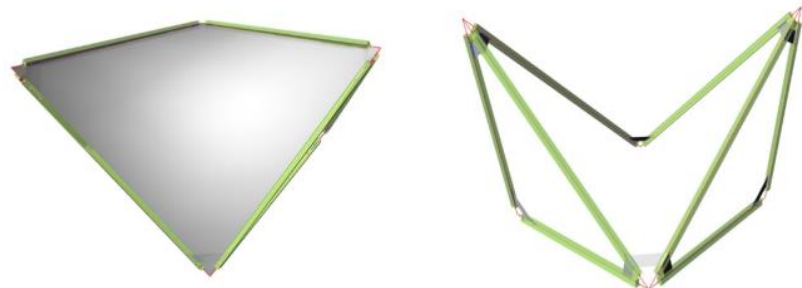

Fig. 7: Basic Scheme of the Ruled Surface with the basic skeleton to be robotically fabricated (Image from author)

The ruled surface pavilion is an attempt to produce a structural entity that can be fabricated using a robotic arm in order to optimize time and precision. Such exercise could be made manually, but due to the distinct angles of cut, it would require an exceeding amount of time in addition to risking the precision of the outcome. In addition, machines such as the $\mathrm{CNC}$ or the laser cutter would not be able to perform the job, thus leaving the options reduced to the use of the robotic arm.

The construction strategy was based on defining a standard material to be dealt with and matching the proper aesthetics with the fabrication procedures and the restrictions of the machines to be used. The fabrication of the components would be as simple as positioning the beams on a table, and with a simple definition to 
be sent for fabrication. However, the relation among the robotic arm constraints, tool capabilities and the raw material characteristics would have to be taken into consideration. Special fixations on the table were in this case set up so that the beams would be properly fixed to the working table. In addition, and due to the larger dimensions of the beam in comparison to a standard cutting table, flexibility was allowed so that the beams could be placed according to the reach of the robotic arm.

A standard wooden beam of a $5 \mathrm{~cm} \times 7 \mathrm{~cm}$ cross section was chosen to define the basic structure of the pavilion. The edges of the surface would be fabricated using such material. Therefore, what needed to be dealt with in the design process was the way in which the beams would be connected, taking into consideration that each beam would have a different length.

A definition was created in Grasshopper KUKA/prc in order to realize straight inclined cuts on wooden beams that would serve as slots that would later connect with other beams by a 2D cut wooden panel and fit according to the proper orientation of the beams. The procedure consisted in intersecting the wooden connectors with the beams, which would result into two straight but inclined lines that would later guide the inclination of the tool tip attached to the robotic arm, its distance, and the depth of cut. In addition, such lines would help in defining the relation between the wooden beam and the tool tip with suitable planes and step-down distances between each cut until reaching the bottom of the intersection, thus allowing multiple passes instead of one single cut. Since the tool used had a $20 \mathrm{~mm}$ diameter, only the depth equivalent to the diameter would be executed in order to avoid damage of the tool "Fig.8".

After robotically cutting the slots, the beam would then be divided in equal number in all sides and later divided in an equidistant manner in order to attach the hooks for the wire to be later distributed in order to define the inner part of the ruled surface. Therefore, each wooden beam would have at least two different inclined cuts that, with the next beam, would be connected with the flat wooden articulation.

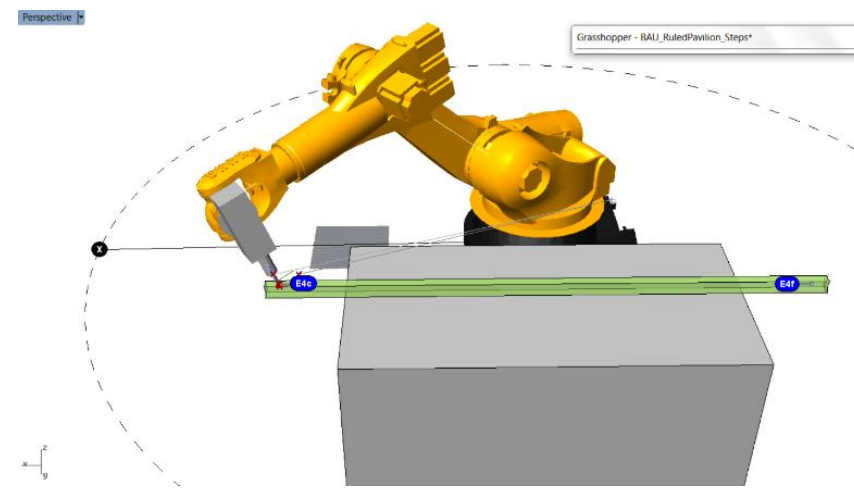

Fig. 8: Simulation of the fabrication process taking into consideration the relation among material, machine and tool (Image from author)

Physical conditions of the tools used were taken into consideration in order to adapt them with the assembly process. Basically, the beams were cut with a $20 \mathrm{~mm}$ tool bit attached to a spindle in the robotic arm. This implied first in revising the angles of cut in relation to the physical condition of the tool in order to avoid collision with the material and to ensure that the tool can reach the desired depths.

In order to execute a cut respecting the constraints of both machine and material, a series of planes were defined in the space of cut in order to first control the angle of approach of the tool bit, and second to realize the cut in a series of step down procedure. In addition, and since the length of the beams were considerably large, a strategy had to be defined in order for the robotic arm to reach the place of cut and execute the job, with the idea that the beam would be rotated several times in order for the job to be executed at each side of the beam requiring pocketing.

A total of 8 beams and 12 connectors have been fabricated in approximately 18 minutes. Optimization in time has been met in accordance to the objectives, even though the assembly of the pavilion took close to 5 hours "Fig.9". However, due to the inclinations and numbering of the components, assembly was relatively easy, with the exception of attaching the hooks on beams in order to extend the mesh throughout them to form the parabolic surface. Despite this fact, the main challenge in such an exercise was to define a fabrication strategy that only a machine with more than 3 axis would be able to execute, in addition to providing possibilities of optimization of time and precision.

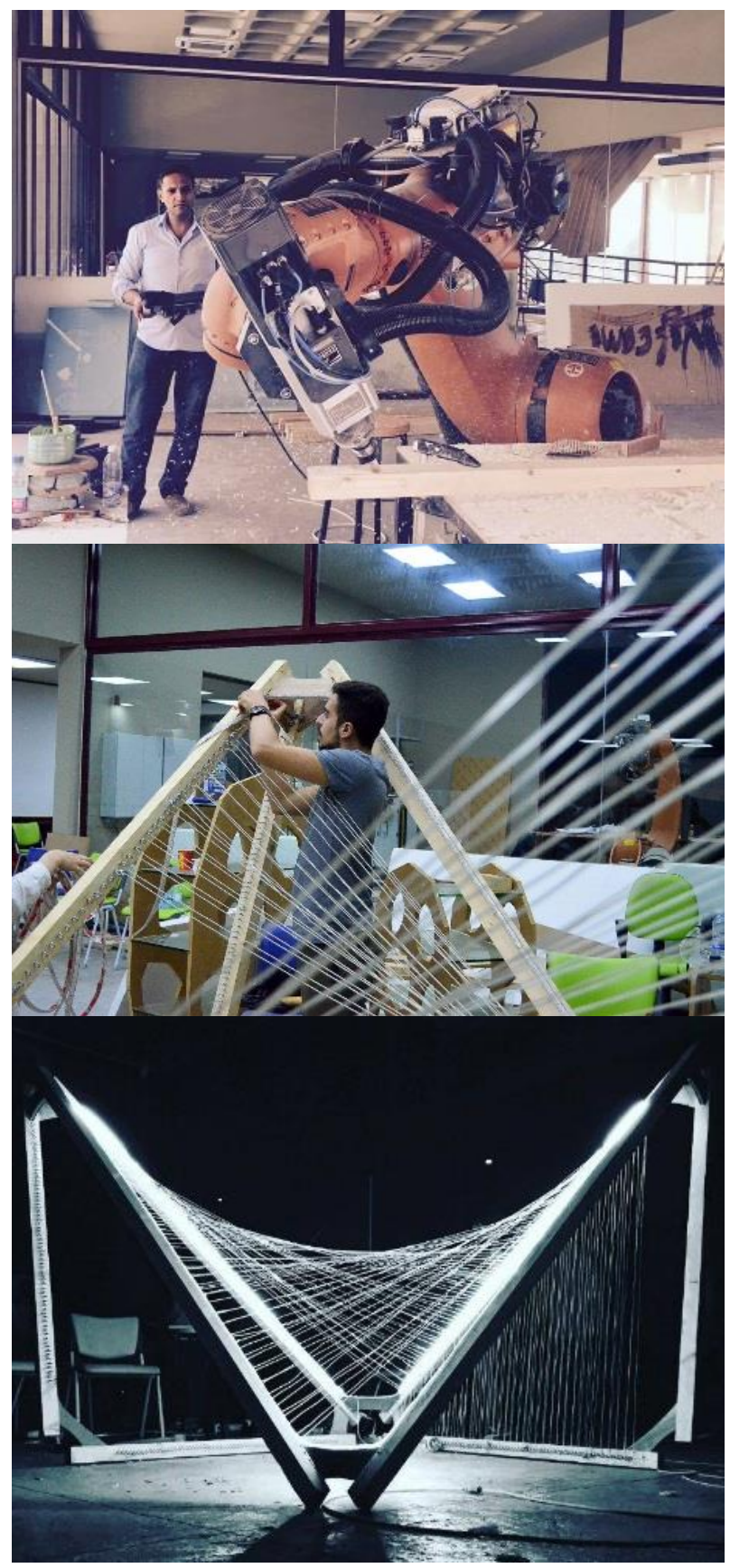

Fig. 9: Final Structure after assembly (Image from author)

\section{Conclusion}

Within this paper, three experiments related to digital design and production of architecture have been presented. Undertaken investigations range from the research of new geometric architectural 
design methods to optimizations of integrated manufacturing techniques. The spectrum of the research has been spread in order to provide a global approach to the problem of design and production of architectural free-form objects. It is highly important to situate the links of the digital chain within the global context of the design process engaged.

The applications presented show how beneficial and time-saving the use of a complete design chain can be, allowing to intervene independently at any of the design or production steps and permitting changes within the design process to be integrated smoothly. The initial appraisal, claiming the lack of feasibility of free-form structures shows the importance of the optimization of design and production processes involved within the realization of architectural free-form objects. In addition, the generation of the data describing the constructional components of iterative geometric objects raised methods for data structuring, thus allowing the set of geometric data to be conditioned for processing throughout integrated manufacturing.

\section{References}

[1] Y. Weinand, Advanced timber structures. Architectural designs and Digital dimensioning. Basel, Birkhäuser, 2017.

[2] H. Markus, Origami-Folded Plate Structures. PhD Thesis N. 4714 École Polytechnique Fédérale De Lausanne, 2010.

[3] I. Stotz, Iterative geometric design for architecture. $\mathrm{PhD}$ Thesis $\mathrm{N}$. 4572 École Polytechnique Fédérale De Lausanne, 2009.

[4] H.U. Buri, Timberfabric - Applying Textile Assembly Principles for Wood Construction in Architecture. PhD Thesis N. 5553 École Polytechnique Fédérale De Lausanne, 2013.

[5] J. Bonet, L'Últim Gaudi: El Modulat Geomètric del Temple de la Sagrada Família, 2nd ed., Barcelona: Pòrtic, 2001, pp. 42-104.

[6] W. L. Edge, The Theory of Ruled Surfaces, New York: Cambridge University Press, pp. 11-14. 